\title{
Exploring the diverse, intimate lives of plants
}

\author{
New ways to assess plant-microbe interactions can yield unexpected paths to biodiversity.
}

\section{Vivien Marx}

\section{/ /}

've invented a new algorithm to predict phenotype from genotype," says the presenter in a cartoon about a lab meeting. "Brilliant!" "Superb!" exclaim the attendees. Says another meeting attendee, "Someone did it in plants in 1978." In next cartoon panel, that last commenter is tossed out a window. Scientific findings in animals are sometimes "hailed as breakthroughs" when in fact they were predated by discoveries in plants, as in the case of RNA interference and immune receptors, notes ${ }^{1}$ Nick Talbot, who directs the Sainsbury Laboratory in Norwich, UK, where he also runs a lab. Plant blindness, as the underappreciation of plants is sometimes called, is an eye-roll-inducing evergreen. A number of plant labs develop and use new approaches to assess how plants, fungi and microbes interact. There's more than give and take between plants and microbes; dramatic, intimate strategies are at work $^{2-6}$. Given that climate change demands attention, a deep understanding of these interactions offers new ways to address how to sustain ecosystems and biodiversity. "We are in a new era that puts networks in the center of our understanding of biodiversity," says Toby Kiers, an evolutionary biologist at Vrije Universiteit Amsterdam.

\section{Below ground}

"While the spotlight has largely focused on human gut microbiomes, plant roots house one of the most diverse, yet vastly neglected, microbial communities on Earth," says Kiers. Plants roots are covered by fungi that build mycorrhizal networks underground: long, thready webs that integrate information. The fungi deliver nutrients to plants, receive nutrients in return and connect plants to one another. Biodiversity is often seen as a static measure of species numbers. Instead, Kiers and her colleagues look at the diversity of strategies, such as those that shape the interaction of plants and microbes, especially fungi. "Fungi are incredible in this way: you can expose them to a new situation or environment and track their growth and trade strategies," she says. "We are just starting to understand how a microbial strategy - for example whether a particular member of a community functions as a 'mutualist' or an 'antagonist'-

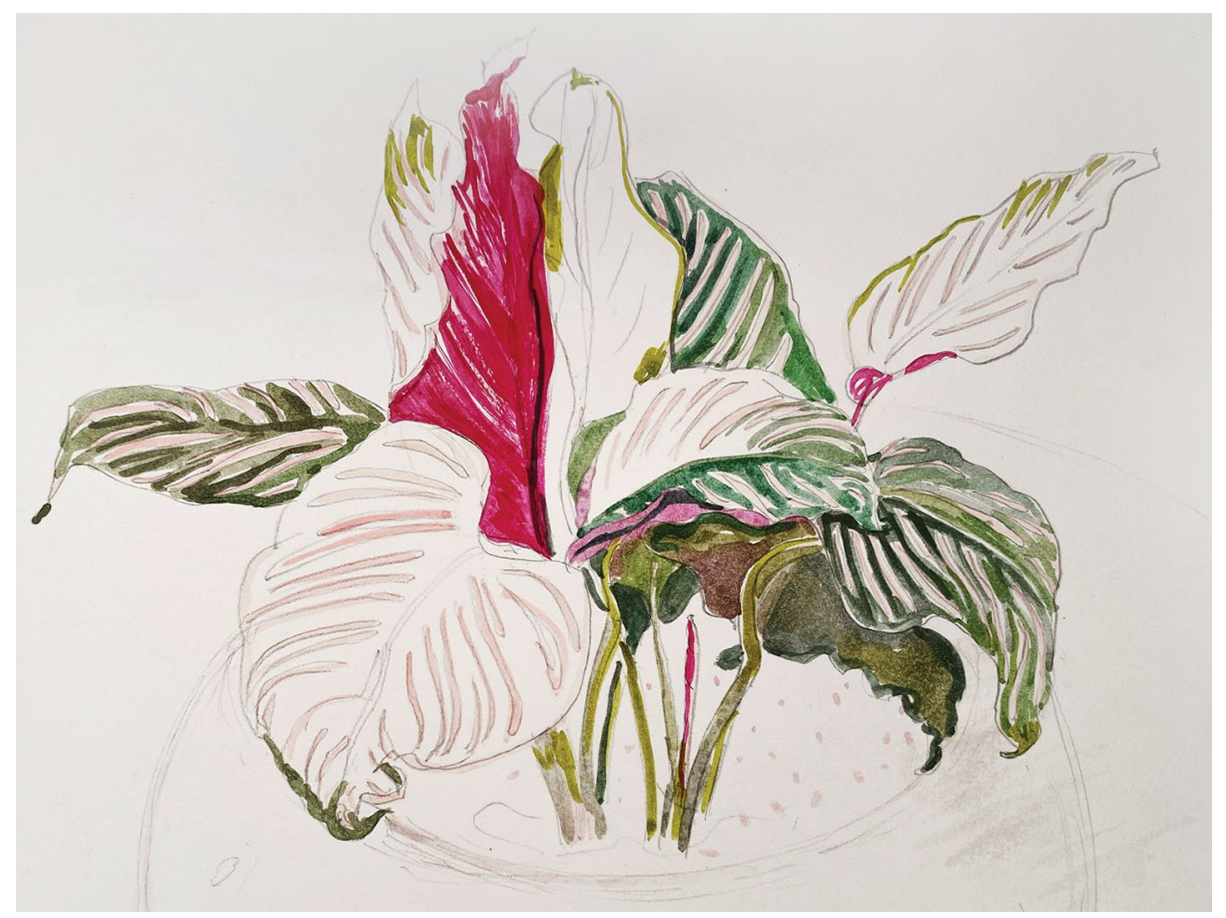

Some plant labs work on new ways to characterize how plants, fungi and other microbes interact. Such research can help to address climate change and sustain ecosystems and biodiversity. Credit: E. Dewalt

depends on the presence or absence of other microbes," says Kiers. When a microbe is the only member of a consortium, it may be more pathogenic and colonize all of a plant or a plant community's roots. "However, if it is competing with other microbes in a community, it may act more as a commensalist or even a mutualist."

Fungi can be bad news for plants. Talbot studies the fungus Magnaporthe oryzae, which leads to the rice blast that brings on periodic epidemics and devastating crop loss in China, Korea, Japan and the United States. Regine Kahmann from the Max Planck Institute for Terrestrial Microbiology in Marburg, Germany, has long studied a corn pathogen, the fungus Ustilago maydis. This fungus stunts growth of the corn plants it infects but does not kill. She and her team recently presented findings ${ }^{7}, 16$ years in the making, showing that a complex of seven proteins is critical for the fungus's virulence. "It really establishes, throughout the life cycle inside the plant, a very intimate relationship in which the fungus needs to make sure that this goddamn plant stays alive, because only then can the plant provide the nutrients the fungus needs," she says.

Fungi helped ancestral plants launch their trek from Earth's watery habitat to life on land around 600 million years ago. There are slow-burning disputes about the details of this journey from water by "little more than puddles of green tissue" onto land, as Merlin Sheldrake points out ${ }^{5}$. Sheldrake is a biologist, author and research associate in Kiers's lab at Vrije University. But there's agreement that this alliance evolved into an intimate collaboration between fungi and plant root systems. Just about all organisms on land benefit from these relations, as plants form the base of the food chain.

With mycorrhizae, plants have food providers underfoot. Fungi build a dense, branched network that can dwarf the plants' own roots. The fungi forage for phosphorous, nitrogen, zinc and copper 
from soil and offer them to plants. The plants give the fungi nutrients such as sugars and lipids produced through photosynthesis. Under the microscope, in samples of roots that have been boiled in dye and squashed onto a glass slide, there's "an intertwining," notes Sheldrake. "Fungal hyphae fork and fuse and erupt within plants cells in a riot of branching filaments. Plant and fungus clasp one another. It's difficult to imagine a more intimate set of poses." Like most intimate relations, these are entangled, complex and diverse. When I asked Sheldrake how junior scientists building their trajectory might study such plant-microbial relations, he says much depends on the ecosystem and angle a researcher will want to take. But, where possible, plant researchers will want to heed "what's happening below ground."

Indeed, says Melanie-Jayne Howes, who leads research in phytochemistry and pharmacognosy at the Royal Botanic Gardens, Kew, there's huge diversity underground but also in the air, the sea and all around us, which opens up a way to study diversity and seek new solutions to some of our global challenges. Advances such as DNA sequencing technologies enable this quest, she says, helping scientists uncover the vast interactions between organisms.

\section{Herbaria bounty}

Planet Earth is home to around 350,000 plant species, 325,000 of which are flowering plants. An estimated two in five plants are threatened with extinction ${ }^{8}$. The UN Sustainable Development Goal No. 15 calls for protecting terrestrial ecosystems and halting biodiversity loss. "However, we can't assess how threatened a species is until we know it exists, " note the authors of a Kew report.

Every year around 4,000 new species of plants and fungi are scientifically named for the first time, says Howes. Even as scientific progress is made with known species, "along come another few thousand for us to understand their role in ecosystems," says Howes. One newcomer species is Artemisia baxoiensis in Tibet, a close relative of the Artemisia annua that is a source of the antimalarial drug artemisinin. Another recent discovery is Galanthus bursanus. A cousin Galanthus species is a source of galantamine, which is used to treat some dementia symptoms. Studying species new to science, she says, could reveal potential new medicines and "could help identify new optimum sources of them, which are potentially less damaging to the environment."

Of the 350,000 known plant species, seven percent have documented medicinal uses, she says. Conservation biologists

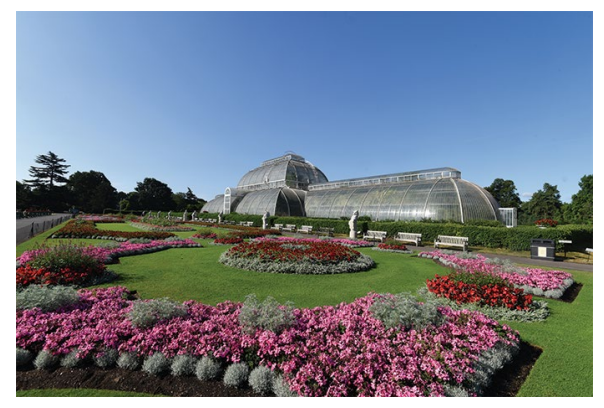

A team from the Royal Botanic Gardens Kew, including Howes and colleagues, sees great promise in a comprehensive scientific study of the world's botanic gardens, herbaria, seed banks and fungal collections. Here, Kew's Palm House. Credit: J. Eden/RBG Kew

have long thought about how to widen this potential. A team from the Royal Botanic Gardens Kew, including Howes and others, and colleagues from several institutions have a proposal ${ }^{9}$ : a comprehensive scientific study of biodiversity that could inspire, accelerate and innovate the hunt for pharmaceuticals derived from plants and fungi. The group, says Howes, represents the expertise needed for such an enterprise: chemistry, genomics, taxonomy, drug discovery, botany, mycology.

Before anyone feels ready to pack their bags to help out, reality butts in. Beyond travel limitations in a lingering pandemic, the fieldwork to obtain usable plant and fungal material takes time and money. And one cannot just take plants and fungi from nations and Indigenous peoples. Botanic gardens maintain around one-third of all known land plant species, herbaria hold around 380 million specimens and fungal collections hold around 860,000 strains. Such samples are already being used in genomic research and to inform conservation efforts, given how rich a species record across the globe and through time and space it offers. The group's idea is to analyze select species from the world's botanic gardens, herbaria, seed banks and fungal collections and reckon with the heritage of specimens, such as those obtained during times of colonial rule and removed without consent or involvement of local inhabitants.

Chemical analysis of these collections sounds tempting but destructive. But, says Howes, it has become possible to analyze the chemistry of herbarium specimens without damaging them. Only a few milligrams are needed to apply a method that involves liquid chromatography with photodiode array detection and high resolution mass spectrometry. For analyzing the chemical compositions of plants and fungi as they hunt for medicinal molecules, Howes also prizes technical advances such as cryo-electron microscopy and microcrystal electron diffraction, as well as advances in nuclear magnetic resonance spectroscopy and mass spectrometry imaging.

A better understanding of the evolutionary relationships between species comes from phylogenetics research, underpinned by advances in DNA sequencing, says Howes. This in turn helps predict which plants and fungi might produce medicinal or other useful compounds and sheds light on the most sustainable species sources. Insight into biosynthetic pathways in plants and fungi can open up ways of expressing these pathways in organisms such as yeast, which can boost yields and reduce a need for wild harvests of a given plant or fungus. The chemical analysis of this vast number of samples can provide insights into their chemical diversity and unlock a trove of potential medicines and, says Howes, "enable us to identify more sustainable alternatives to source medicinal or other useful chemicals, helping to prevent biodiversity loss."

\section{Microbial punch}

Preventing biodiversity loss and addressing sustainability necessitates a better understanding of plant pathogens. What fascinates Talbot most about destructive plant pathogens such as M. oryzae is how their complex developmental process lets them breach plant cells and gain entry to plant tissue while actively suppressing plant immunity. The fungus uses specialized infection structures called appressoria deployed by many disease-causing microorganisms ${ }^{10}$. In the case of $M$. oryzae,

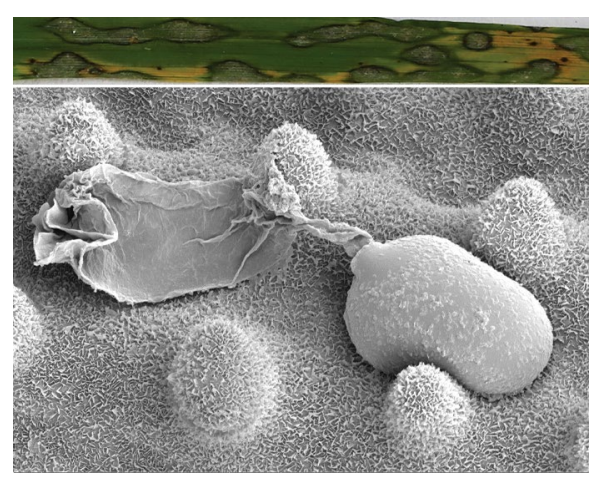

Some fungi cause massive damage. M. oryzae causes rice blast. An electron micrograph shows its appressoria, the fungal infection structures, punching their way into a rice leaf. Credit: $L$. Ryder/The Sainsbury Laboratory; K. Findlay/John Innes Centre 
the fungus adheres to the surface the leaf of a rice plant and, through enzymes and physical force, pushes its way into a plant cuticle, infecting the plant. Push is phrasing it mildly: it punches rice leaves with a pressure wallop of 80 atmospheres, around 40 times a typical car tire pressure. This power stems from the osmotic pressure of accumulated solutes such as glycerol. The infection spreads throughout the plant and spreads from one rice plant to the next through wind and splashing dew drops.

For researchers working on plant disease and plant immunity, microbiome knowledge changes their perspective, says Talbot. The plant microbiome includes consortia of endophytic bacteria and filamentous microbes such as fungi. There's the mutually beneficial plant-mycorrhizal interaction and, separately, plants have sophisticated immune receptors to prevent disease-causing organisms from invading plant tissue. "The relationships are more complex, however, than previously thought," he says. In his view, single-cell techniques used in labs to assess gene expression, proteomics and metabolomics are set to dramatically change the understanding of microbial interactions with plants. "By comparing these to existing tissue or whole organism-level datasets, we will begin to appreciate the temporal and spatial dynamics of plant-microbe interactions in unparalleled detail," he says. "This is a really exciting prospect."

The seven-protein complex that Kahmann and her team uncovered ${ }^{7}$ supplies Ustilago maydis, the corn smut fungus, with its virulence. To infect corn, this fungus also uses appressoria. Rather than a punch, it presses through the plant cuticle and plant cell wall, creating an invagination through which the multi-protein complex can interact with the plant. It suppresses plant immune defenses. The team is still characterizing the stoichiometry of the protein complex, but they have found that all members are expressed during infection and the proteins appear to be co-regulated. While working on this long-term project, Kahmann remembered a paper on the first fungal translocated effector protein. She returned to it and noted similarities to the structures she and her team were seeing in their electron microscopy with immunogold labeling experiments. As it turns out, Ustilago's protein complex is also a virulence mediator in other fungi such as rust, which ravages wheat and other crops, too. For a small molecule screen and to avoid the years it would have taken them to carry out 200,000 maize infections, they reconstituted three of the seven complex members in yeast. The yeast were engineered to grow

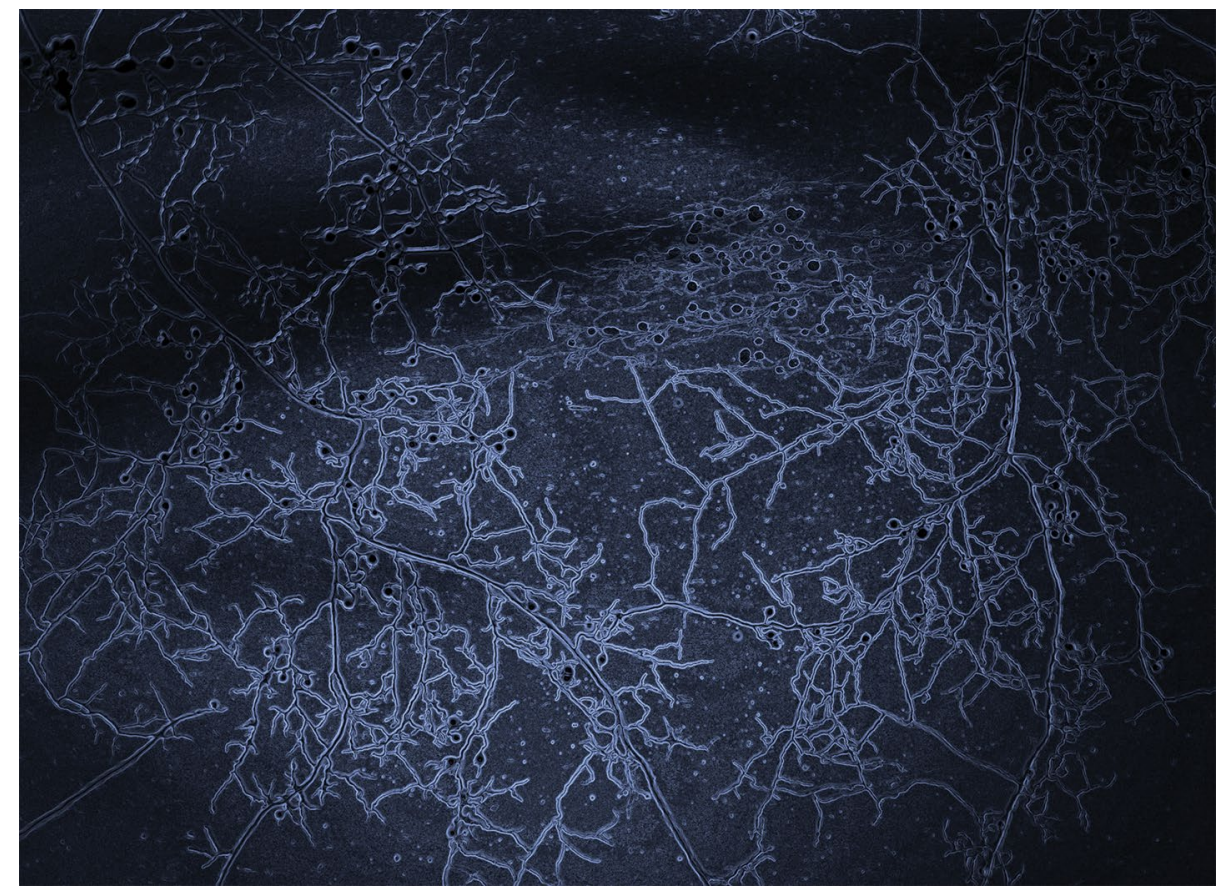

Toby Kiers and colleagues study the plant-microbial underground marketplace. Their imaging robot captures network growth of Rhizophagus aggregatum, a fungus that hoards phosphorous from soil to drive up the price plants must 'pay'. Credit: L. Oyarte Gálvez

only in the presence of this subcomplex. Then they tested several 'hits'. Several small molecules stopped virulence not only of Ustilago in maize but also of Uromyces fabae in beans. "Lo and behold, the compounds also work on rust fungi," she says and bursts out laughing.

After decades of working on a basic research question-understanding how the fungus delivers its effector proteins to the plant-she is now talking to companies about potential licensing agreements for some of the compounds that battle rust. When scientists set out on their trajectory, she advises they "pick something they are really deeply excited about, because otherwise they will not have the strength to go through these valleys where nothing works and where they are simply stuck for a long time." Overall, she sees a trend in basic and applied plant biology to explore how to tweak a plant's microbiome to shape plant health. That's a dive into plant-based decision-making.

\section{Plant strategies}

With sequenced plant genomes, researchers usually take a comparative or evolutionary analytical approach. Kiers and her colleagues study organismal decision-making and build experimental setups to enable this. They might force partners to cheat in a trade deal and study how the other partner reacts. "Of course, there is no cognition, no brain involved," says Kiers. The trade in question is the marketplace of mycorrhizal fungi and plants.

This trade is not one of human economy, but the activities ring familiar: there is exchange, selling and buying, hoarding, begging, borrowing, cheating. Plants supply more carbohydrates to fungal partners that provide more phosphorus to them and vice versa, she says.

Natural selection shapes the microbial strategies that are an overlooked component of biodiversity, she says. Capturing these events is hard. They happen underground and there are loads of confounders. Plants trade only in carbon with fungal partners; fungi can trade multiple resources, such as nitrogen and trace elements. In the lab "it takes achingly precise experiments and careful manipulations to quantify strategies," she says. The benefit is they can learn how microbes integrate a complex array of chemical, physical and environmental stimuli, which gives insight into the rich social lives of microbes and strategy diversity.

In the lab, Kiers and team capture high-resolution time-lapse videos of fungal trade routes. They developed nanoprobes to track resource exchange: they tag a rock phosphate - hydroxyapatite-with quantum dots. When exposed to ultraviolet light, the tagged nutrients fluoresce. This lets the researchers capture a time series of fluorescent nanoparticles in three 
colors. Using confocal microscopy they can quantify, for example, how much phosphorus is being stored in hyphae, the fungi's long branches. With this approach, she says, "we found fungal networks dynamically hoard and transfer resources depending on the nutrient status of the plants connected to network." The researchers want to link plant productivity to fungal network structure and have begun scaling up imaging to generate three-dimensional images of intact fungal networks.

To measure intrahyphal flow and capture the overall physical architecture of fungal networks as they grow on root cultures in vitro, the team has built a high-throughput imaging and high-resolution video capture platform with Loreto Oyarte Gálvez and Tom Shimizu from the AMOLF physics institute in Amsterdam. The system is a remote-controlled imaging robot that delivers time-resolved datasets from more than 30 fungal networks at the same time. Its optical positioning system helps them collect real-time videos of nutrient flows at high resolution at known physical coordinates across the network. Then they can switch to lower resolution to collect data on fungal topology. Across the fungal network's different sectors, the videos show flow taking place at different speeds. And it runs in different directions. Along with Shimizu and Princeton University researchers Howard Stone and Philippe Bourrianne, who study microscale fluid dynamics, they built models of fluid flows at different positions in the network. "Given that these fungal flows are responsible for massive amounts of nutrient transfer across ecosystems and can store huge amounts of carbon, we are trying to link underground flows to aboveground ecosystem dynamics, including how flows influence biodiversity," says Kiers.

In her view, capturing nutrient flow shapes understanding of the biodiversity of microbial strategies. "Our idea is that by oscillating its internal flow, a fungus can compare its nutrient status across and space and time, such that flow can be subsequently redirected," she says. "Because we can now start to track nutrient flows, we can ask whether oscillations help the fungus to regulate, or actively 'calculate', where and when to trade." Fungi use electrical activity to coordinate their responses to stimuli, a bit like neural networks in animals. As the team explores how a fungus integrates information across an immense number of hyphal tips while connected to multiple plants and extended over tens of meters, they want to also focus on aspects such

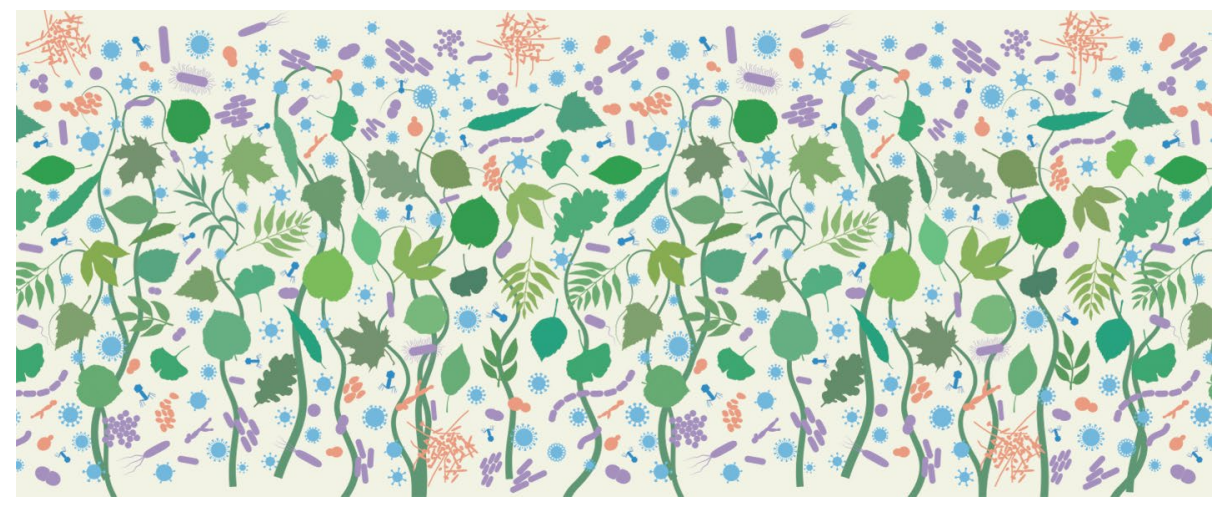

Microbes and plants are intimately intertwined. To study biodiversity and address climate change, scientists assess how species are embedded in complex networks of interactions. Credit: P. Patenall / Springer Nature Limited

as voltage changes across cell membranes that help to coordinate such network-level responses. They plan to use neuroscience tools such as microelectrode arrays to quantify electrical activity in symbiotic networks and stimuli-related changes.

\section{Networked capture}

"Species interactions are context dependent," says Kyoto University researcher Hirokazu Toju, who collaborates with Kiers ${ }^{3}$. Changes in biotic and abiotic environments can alter the strength and direction of species interactions through time. To describe biological systems, one might apply standard approaches, but he finds mathematical approaches more promising that focus on time-series changes of species' interactions in terms of both direction and strength. Theory-based approaches such as empirical dynamic modeling help to provide an understanding of the architecture of species interaction networks and how it changes through time and with environmental conditions.

Machine learning approaches can help to predict growth of fungal networks under different conditions. Kiers and her colleagues use machine learning to analyze the terabytes of data the imaging robot delivers every month. They extract the network skeleton at every node, she says, and follow hundreds of thousands of nodes through time. They can manipulate the network and follow that through those many nodes. Such experiments let the team test big ideas about network formation in nature, under what conditions networks are robust to climate change, how they react to low or high nutrients, and what conditions select for higher efficiencies. "At its core, our research will tell us how microbial strategies are expected to shift under climate change," says Kiers.
Machine learning can be powerful not only for finding keystone species and but also for designing core microbiomes that can support designed systems in agriculture, says Toju, but one needs sufficient microbiome data to distinguish "benign" from "unfavorable" states of microbial communities. To go beyond case-by-case inference or pattern recognition, "theory-based approaches integrating ecology, microbiology, mathematics, and physics are necessary to reveal basic rules underlying ecological community dynamics." Some labs build and use synthetic microbial communities, but in Toju's view, there's no guarantee these work efficiently if the focus is on functions of respective microbial species. For example, in the case of two plant-growth-promoting microbial species, if these two microbes have different sets of genes, "we may expect that co-inoculation of the two microbes will be highly beneficial to host plants," he says. "However, this is not necessarily the case." Co-inoculation effects often diverge greatly from expected effects based on single-inoculation data. Such results mean that, when working on synthetic microbial community approaches, researchers need information on functions of each microbial species and information about mechanisms at the multi-species level, such as the species-interaction network architecture, he says. Given climate change and the emergence of new types of pests and pathogens, scientists can take such knowledge and apply it to support the "entangled webs of interactions" in agroecosystems, where plants, insects and microorganisms including bacteria, archaea, protists and fungi interact, says Toju. He works on plant microbiomes, the human gut microbiome and invertebrate-associated microbiomes. Animals and plants are 
readily observed in nature and in labs, but ecological studies can reach another level now that high-throughput sequencing datasets reveal the compositions of microbial communities. Within microbial systems, there tends to be redundancy, with countless species fulfilling similar functions, he says. Plant and animal communities have less redundancy, and their dynamics are more likely to be affected by the particular traits of constituent species. It's why he expects the principles of ecological processes to be uncovered more clearly in species-rich microbial systems than with only animal or only plant communities. This view led him, around a decade ago, to recast his research focus from animals and plants to microbial communities and those involving both microbes and macro-organismal hosts. "With rich, reliable datasets, ecological studies on microbiomes will dramatically promote understandings of basic rules in animal and plant community dynamics," says Toju.

In ecological research, "microbiomes have another advantage," he says. They regenerate much faster than animals or plants, which is a trait that makes them ideal for understanding the feedback loops between evolutionary and ecological processes, he says. By targeting microbiomes, one can explore the dynamics of qualitative changes, evolutionary genetic change and quantitative changes that affect the number of individuals or cell numbers ${ }^{11}$.

Such theoretical and informatics frameworks are applicable to diverse types of microbial systems, such as gut microbiomes and plant-associated microbiomes, he says. Toju and colleagues have developed a general algorithm for designing "functional core microbiomes" based on information regarding constituent species' functionsthe genomes-and interspecific interaction networks. "I applied the algorithm to three types of microbiomes: namely, mouse gut, crop-plant-associated, and experimental aquatic microbiomes," he says. In addition to the core microbiome design algorithms, he and his colleagues also apply mathematical tools used in nonlinear dynamic analysis to highlight key species interactions that have profound effects on the dynamics of entire microbiomes, he says. They explore how to predict drastic changes in microbial community composition such as those involved in human gut dysbiosis. Common ecological theories and community-design algorithms are applicable to diverse microbial communities, and the ability

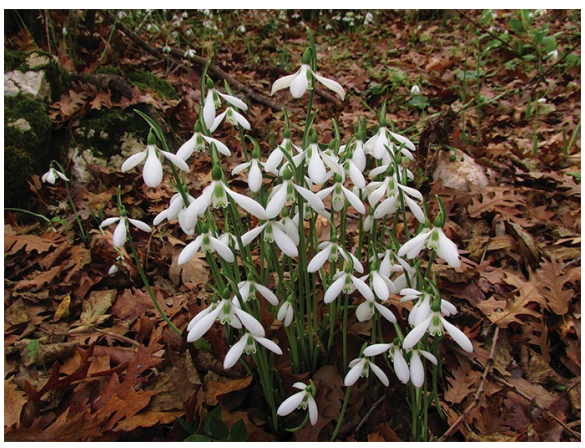

Every year around 4,000 species of plants and fungi are newly described. Shown here, Galanthus bursanus, a cousin of the Galanthus species that yields galantamine. Galantamine is used to treat some dementia symptoms. Credit: D. Zubov

to control microbiomes will vary. $\mathrm{He}$ thinks that controlling plant-associated microbiomes will be easier than controlling human gut microbiomes. What matters in such projects is what he calls "priority effects" in species assembly.

\section{Community thinking}

In ecological communities of microbes and macro-organisms, says Toju, species that colonize new habitats earlier than others have huge advantages since they can physically block following colonizers. They may defend their habitats by producing antibiotics and can stimulate host plantanimal immune responses, which would indirectly hinder colonization by others. Such historical effects are expected to impede shifts among "alternative stable states" of species compositions.

While it remains challenging to change gut microbiomes from community compositions that cause disease to ones associated with healthy guts, plant seeds are nearly devoid of bacteria and fungi. He sees that as an indication that scientists will be able to control microbiome dynamics and functions in agriculture, says Toju. One approach he explores when he designs core sets of microbes to promote microbiome assembly beneficial to plant hosts is that he introduces those microbiomes into seeds or seedlings. The goal is to promote plant growth and increase plants' resistance to biotic and abiotic stresses.

Perhaps the same or similar sets of core microbiomes can be applied to diverse crop plant species. That's possible because the diverse mycorrhizal and endophytic fungi have a broad range of hosts-many of them can form a symbiosis with almost all land plant taxa. In this manner one could alter plant resistance relative to biotic and abiotic environments without modifying plants' own genomes, he says. Designed microbiomes can be a kind of defense system as a quick and low-cost prevention or treatment related to pathogen outbreaks. And they can complement existing plant breeding efforts. In his view, research efforts on plant-associated and animal-associated microbiomes have much to offer one another and they enhance our understanding of systems consisting of multiple species, says Toju. Species are embedded in complex networks of interactions with other species, and these shape many of their strategies, says Kiers. From nested symbioses with plants, in which plants are colonized by fungi and the fungi in turn are colonized by endosymbiotic bacteria, "biodiversity is best described as layers of interacting strategies." Given how intense and fast climate change is proceeding, we need to understand how climate shifts will drive changes in these strategies, she says. "What interactions will break down, what networks will become more robust?" The aim should be to conserve network interactions, not just the species. "Microbes are key to our fight against climate change," says Kiers. They adapt and are faster and more efficient at it than anything humans can do. "What has been missing on the conservation agenda are the organisms we can't see, and all the fantastic strategies they have evolved."

Vivien Marx ${ }^{\bowtie}$
Nature Methods.
$\bigotimes_{\text {e-mail:v.marx@us.nature.com }}$

Published online: 16 July 2021

https://doi.org/10.1038/s41592-021-01228-x

References

1. Talbot, N. Nat. Plants 6, 1068-1069 (2020).

2. Werner, G. D. A. et al. Proc. Natl Acad. Sci. USA 4 1237-1244 (2014)

3. Toju, H. et al. Nat. Plants 4, 247-257 (2018).

4. Genre, A., Lanfranco, L., Perotto, S. \& Bonfante, P. Nat. Rev. Microbiol. 18, 649-660 (2020).

5. Sheldrake, M. Entangled Life: How Fungi Make our Worlds, Change our Minds and Shape our Futures (Penguin Random House, 2020)

6. Trivedi, P., Leach, J. E., Tringe, S. G., Sa, T. \& Singh, B. K. Nat. Rev. Microbiol. 18, 607-621 (2020).

7. Ludwig, N. et al. Nat. Microbiol. 6, 722-730 (2021).

8. Antonelli, A. et al. State of the World's Plants and Fungi (Royal Botanic Gardens (Kew), 2020).

9. Pérez-Escobar, O. A. et al. Science 369, 781-782 (2020).

10. Talbot, N. Curr. Biol. 29, R137-R149 (2019).

11. Toju, H. Nat. Ecol. Evol. 1, 0024 (2017). 